\title{
Dynamics at infinity and a Hopf bifurcation arising in a quadratic system with coexisting attractors
}

\author{
ZHEN WANG ${ }^{1,2, *}$, IRENE MOROZ ${ }^{3}$, ZHOUCHAO WEI $^{3}$ and HAIPENG REN ${ }^{2}$ \\ ${ }^{1}$ School of Science, Xijing University, Xi' an 710123, China \\ ${ }^{2}$ Shaanxi Key Laboratory of Complex System Control and Intelligent Information Processing, \\ Xi' an University of Technology, Xi' an 710048, China \\ ${ }^{3}$ Mathematical Institute, University of Oxford, Oxford OX2 6GG, UK \\ *Corresponding author. E-mail: williamchristian@163.com
}

MS received 15 February 2017; revised 11 July 2017; accepted 4 September 2017; published online 27 December 2017

\begin{abstract}
Dynamics at infinity and a Hopf bifurcation for a Sprott E system with a very small perturbation constant are studied in this paper. By using Poincaré compactification of polynomial vector fields in $R^{3}$, the dynamics near infinity of the singularities is obtained. Furthermore, in accordance with the centre manifold theorem, the subcritical Hopf bifurcation is analysed and obtained. Numerical simulations demonstrate the correctness of the dynamical and bifurcation analyses. Moreover, by choosing appropriate parameters, this perturbed system can exhibit chaotic, quasiperiodic and periodic dynamics, as well as some coexisting attractors, such as a chaotic attractor coexisting with a periodic attractor for $a>0$, and a chaotic attractor coexisting with a quasiperiodic attractor for $a=0$. Coexisting attractors are not associated with an unstable equilibrium and thus often go undiscovered because they may occur in a small region of parameter space, with a small basin of attraction in the space of initial conditions.
\end{abstract}

Keywords. Infinity dynamics; Hopf bifurcation; coexisting attractors.

PACS No. 05.45.Pq

\section{Introduction}

In 1963, Lorenz [1] showed that a continuous 3D autonomous system of ODEs gave rise to the first chaotic attractor. Chen and Ueta followed this with the Chen attractor in 1999 [2]. Based upon a classification condition given by [3] (see also [4]), the Lorenz and Chen systems satisfy $a_{12} a_{21}>0$ and $a_{12} a_{21}<0$ respectively. Here $a_{12}$ and $a_{21}$ refer to two of the off-diagonal entries in the Jacobian matrix of linearisation about the trivial equilibrium state. A natural question to ask is whether one can construct a chaotic 3D system with $a_{12} a_{21}=0$. We call such a system a transitional system. Fortunately, in 2002, Lü and Chen found a system, which they named as the Lü chaotic system, which satisfied $a_{12} a_{21}=0$ [4]. In addition, in 2008, Yang et al gave another classification condition for chaotic systems [5]. According to this condition, chaotic systems are classified as the Lorenz system group if $a_{11} a_{22}>0$, the Chen system if $a_{11} a_{22}<0$ or the Yang-Chen transitional system group if $a_{11} a_{22}=0$. (For the Lorenz system $a_{11}=-\sigma$ and $a_{22}=-1$.) With the wide applications of chaotic systems in the field of engineering, as well as the mathematical, physical and chemical sciences etc., searching for new chaotic attractors and constructing simple chaotic systems have been intensively considered in the past four decades. Many classical Lorenz-like chaotic systems (of hyperbolic-type) have one saddle and two unstable saddle-foci, and the existence proof of chaos is a common Shilnikov criterion [6]. However, for non-hyperbolic-type chaotic systems, saddle-focus equilibrium does not exist, and the Shilnikov criteria will be invalid in these systems because of the violation of the Shilnikov condition for chaotic systems with stable equilibria. Therefore, it is interesting to ask whether or not 3D autonomous chaotic systems exist with stable equilibria.

In 2008, Yang constructed another chaotic system with one saddle and two stable node-foci, which was further analysed in refs [5-7]. Moreover, another chaotic system with only two stable node-foci is given in 2010 by Yang et al [5]. For a generic 3D quadratic autonomous system, we raise the question: does there exist chaotic system with no equilibrium, one 
equilibrium or any number of equilibria? Sprott [8-10] gave some simple chaotic systems with no equilibrium or one equilibrium by a computer search. Subsequently, chaotic systems with one stable equilibrium [11-15], no equilibrium [16,17], any number of equilibria [18] and a line of equilibrium states [19] have been found and analysed. In the category of chaotic attractors, either self-excited or hidden [20-22], we call chaotic attractors in dynamical systems with no equilibria or with only stable equilibrium hidden attractors. From a computational point of view these hidden attractors cannot be easily found by numerical methods. Furthermore, the knowledge of equilibria does not help with locating hidden attractors [18,23-25]. Therefore, understanding the local and global behaviours of chaotic systems with hidden attractors is of great importance and creating a simple non-hyperbolic-type chaotic system is an important issue for scientists.

For non-hyperbolic type of chaos, saddle-focus equilibrium typically does not exist in the systems. In ref. [8], several non-hyperbolic chaotic systems were proposed by Sprott. One can see that Sprott E system degenerates, and the corresponding equilibria are not stable. It is also easy to imagine that a tiny perturbation to the system may be able to change such a degenerate equilibrium to a stable one. Therefore, we can add a simple constant control parameter to Sprott E chaotic system, trying to change the stability of its single equilibrium to a stable one while preserving its chaotic dynamics. From this point of view, it is easy to understand and indeed easy to prove that the new system will not be topologically equivalent to the Sprott E system. Moreover, local bifurcation such as Hopf bifurcation is the first to be studied with the variation of parameter. However, it cannot obtain global dynamical behaviour generally. To research the global structure of the system, especially in high-dimensional systems, most people will use numerical calculation, but the global structure is not available. So in order to analyse the global dynamics system, we can use the theory of Poincaré compactification, reduce the dimensions of the system on a Poincare ball, and then get the infinity dynamics of the system. Thus, we can obtain the global dynamics structure further. In this paper, motivated by $[11,12,26]$, we study a simple 3D autonomous chaotic system deriving from Sprott Case E system, by adding a small parameter $a$ :

$\dot{x}=y z+a, \quad \dot{y}=x^{2}-y, \quad \dot{z}=1-4 x$.

The paper is organised as follows. In $\S 2$, the stability of equilibria and behaviour at infinity in Poincaré sphere are analysed. In order to obtain a periodic solution, the existence of Hopf bifurcation parameters are determined in $\S 3$. In $\S 4$, the stability of the bifurcating solution is discussed, based on the centre manifold theorem. To verify the theoretical analysis, numerical simulations are given in $§ 5$. Finally, concluding remarks are given in $§ 6$.

\section{Analysis of the dynamics}

\subsection{Basic dynamics}

\section{PROPOSITION 1}

The system (1) possesses only one equilibrium point $P\left(\frac{1}{4}, \frac{1}{16},-16 a\right)$. It is a saddle for $a \leq-0.17903$, either a saddle-focus or a stable node-focus for $a>$ -0.17903 .

Proof. Under the linear transformation $x=X+\frac{1}{4}$, $y=Y+\frac{1}{16}, z=Z-16 a$, system (1) can be transformed into

$\dot{X}=Y Z-16 Y a+\frac{1}{16} Z, \quad \dot{Y}=X^{2}+\frac{1}{2} X-Y, \quad \dot{Z}=-4 X$.

By linearising (2) at the equilibrium point $O(0,0,0)$, the Jacobian matrix is

$J=\left(\begin{array}{ccc}0 & -16 a & 1 / 16 \\ 1 / 2 & -1 & 0 \\ -4 & 0 & 0\end{array}\right)$,

and the corresponding characteristic polynomial is

$f=\lambda^{3}+\lambda^{2}+\left(8 a+\frac{1}{4}\right) \lambda+\frac{1}{4}$.

From ref. [27], when

$a=-\frac{a_{0}}{192}-\frac{55}{48 a_{0}}-\frac{1}{48} \doteq-0.17903$,

$a_{0}=\sqrt[3]{3988+276 \sqrt{69}}$,

the characteristic polynomial has a root of multiplicity 2 and all its roots are real, the equilibrium $O$ is a saddle. When $a<-0.17903$, the characteristic polynomial has three distinct real roots, one is negative, the others are positive, the equilibrium $O$ is a saddle. When $a>-0.17903$, the characteristic polynomial has one real root and two non-real complex conjugate roots, the equilibrium $O$ is a saddle-focus or a node-focus.

\subsection{Dynamics near infinity}

In order to study the behaviour of the trajectories of system (1) near infinity, we shall use the theory of Poincaré compactification in $R^{3}$ in refs [28-30]. Define the Poincaré ball

$$
S^{3}=\left\{r=\left(r_{1}, r_{2}, r_{3}, r_{4}\right) \in R^{4} \mid\|r\|=1\right\}
$$


as the unit sphere,

$S_{+}=\left\{r \in S^{3}, r_{4}>0\right\}, \quad S_{-}=\left\{r \in S^{3}, r_{4}<0\right\}$

as the northern and southern hemispheres respectively. Denote the tangent hyperplanes at the points $( \pm 1,0,0,0),(0, \pm 1,0,0),(0,0, \pm 1,0),(0,0,0, \pm 1)$ by the local chart $U_{i}, V_{i}$ for $i=1,2,3,4$, where $U_{i}=\left\{r \in S^{3}, r_{i}>0\right\}, V_{i}=\left\{r \in S^{3}, r_{i}<0\right\}$. We only consider the local chart $U_{i}, V_{i}$ for $i=1,2,3$ for obtaining the dynamics at $x, y$, and $z$ infinity respectively.

The local charts $U_{1}$ and $V_{1}$

With the change of variables $(x, y, z)=\left(w^{-1}, u w^{-1}\right.$, $v w^{-1}$ ) and $t=w \tau$, system (1) becomes

$$
\left\{\begin{array}{l}
\frac{\mathrm{d} u}{\mathrm{~d} \tau}=1+\frac{w}{2}-w u-u^{2} v+16 a u^{2} w-\frac{u v w}{16} \\
\frac{\mathrm{d} v}{\mathrm{~d} \tau}=-4 w-u v^{2}+16 a u v w-\frac{v^{2} w}{16} \\
\frac{\mathrm{d} w}{\mathrm{~d} \tau}=-u v w+16 a u w^{2}-\frac{v w^{2}}{16}
\end{array}\right.
$$

When $w=0$, system (3) reduces to

$\frac{\mathrm{d} u}{\mathrm{~d} \tau}=1-u^{2} v, \quad \frac{\mathrm{d} v}{\mathrm{~d} \tau}=-u v^{2}$.

System (4) has no equilibrium points, but we can still use a geometrical approach to determine the phase portrait by considering the signs of $\mathrm{d} u / \mathrm{d} \tau$ and $\mathrm{d} v / \mathrm{d} \tau$. When $(\mathrm{d} u / \mathrm{d} \tau)=0$ and $(\mathrm{d} v / \mathrm{d} \tau)=0$, we obtain three nullclines [31]: $v=1 / u^{2}, u=0, v=0$. These nullclines divide the plane into six regions as shown in figure 1. We can also find the general directions of the vector field in these regions and whether the vector field points up, down, to the right, or to the left as in figure 1 .

We can obtain some trajectories in the local phase portraits as shown in figure 2 . The flow in the local chart $V_{1}$ is the same as that in the local chart $U_{1}$, reversing time. Hence, the phase portrait of (1) on the sphere at infinity is at the negative end point of the $x$-axis as shown in figure 2 , reversing the time direction.

The local charts $U_{2}$ and $V_{2}$

Next, we study the dynamics of system (1) at infinity along the $y$-axis. Under the transformation $(x, y, z)=$ $\left(u w^{-1}, w^{-1}, v w^{-1}\right)$, with $t=w \tau$, system (1) becomes

$$
\left\{\begin{array}{l}
\frac{\mathrm{d} u}{\mathrm{~d} \tau}=v-16 a w+\frac{v w}{16}-u^{3}-\frac{u^{2} w}{2}+u w \\
\frac{\mathrm{d} v}{\mathrm{~d} \tau}=-4 u w-v u^{2}-\frac{u v w}{2}+v w \\
\frac{\mathrm{d} w}{\mathrm{~d} \tau}=-w u^{2}-\frac{u w^{2}}{2}+w^{2}
\end{array}\right.
$$

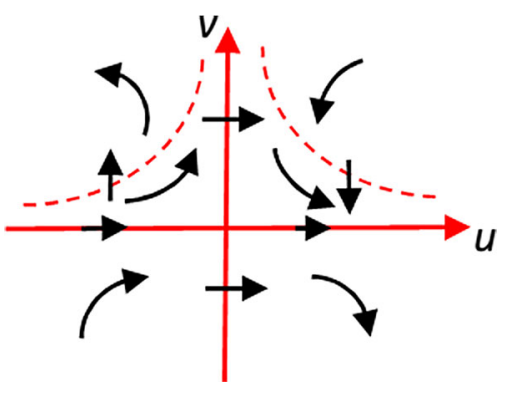

Figure 1. Nullclines for system (4).

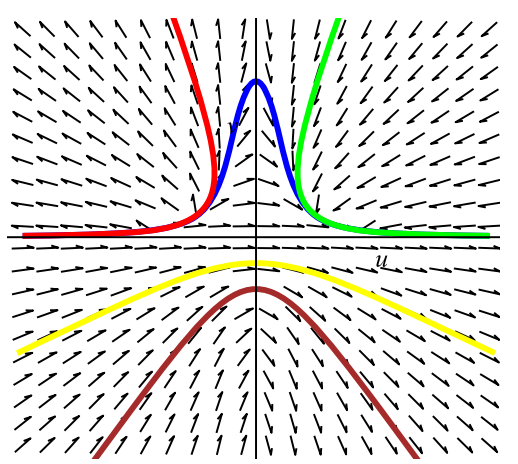

Figure 2. Trajectories in the local phase portraits of system (4).

If $w=0$, system (5) reduces to

$\frac{\mathrm{d} u}{\mathrm{~d} \tau}=v-u^{3}, \quad \frac{\mathrm{d} v}{\mathrm{~d} \tau}=-v u^{2}$,

which has a nilpotent singular point at $(0,0)$. Let $\hat{r}=$ $v-u^{3}$. Then (6) becomes

$\dot{u}=\hat{r}, \quad \dot{\hat{r}}=-u^{5}-4 r u^{2}$.

By the nilpotent singular point theorem [28], we know that the singular point $(0,0)$ is an attracting node. Using the geometrical approach, we can determine the trajectory structure near the origin as shown in figure 3. Also, we can obtain the local phase portraits as shown in figure 4. The flow in the local chart $V_{2}$ is the same as that in the local chart $U_{2}$. Hence, the phase portrait of system (1) on the sphere at the negative infinite end point of the $y$-axis is shown in figure 4 , reversing the direction of time.

The local charts $U_{3}$ and $V_{3}$

Finally, we consider infinity in the $z$ direction. Let $(x, y, z)=\left(u w^{-1}, v w^{-1}, w^{-1}\right)$ and $t=w \tau$. System (1) becomes

$$
\left\{\begin{array}{l}
\frac{\mathrm{d} u}{\mathrm{~d} \tau}=v-16 a w v+\frac{w}{16}+4 u^{2} w \\
\frac{\mathrm{d} v}{\mathrm{~d} \tau}=u^{2}+\frac{w u}{2}-w v+4 u v w \\
\frac{\mathrm{d} w}{\mathrm{~d} \tau}=4 u w^{2}
\end{array}\right.
$$






Figure 3. Nullclines for system (6).

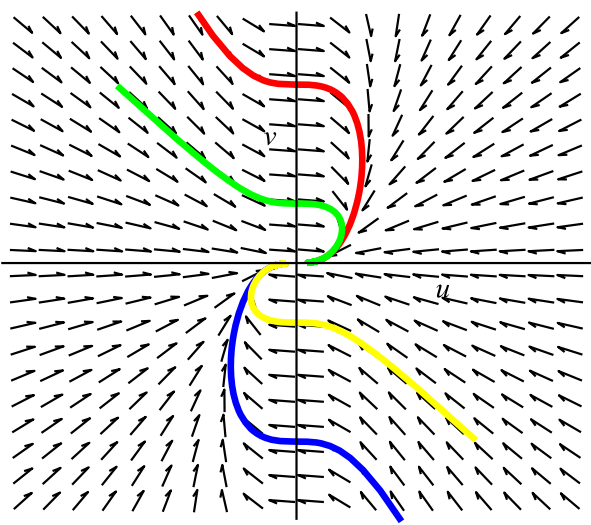

Figure 4. Attracting nilpotent node of system (6).

When $w=0$, system (7) reduces to

$\frac{\mathrm{d} u}{\mathrm{~d} \tau}=v, \quad \frac{\mathrm{d} v}{\mathrm{~d} \tau}=u^{2}$,

with a nilpotent singular point at $(0,0)$. Again, by the nilpotent singular point theorem, we know that $(0,0)$ is a cusp. By computing an energy function, we see that system (8) is a Hamiltonian system, with total energy $E=\frac{1}{2} v^{2}-\frac{1}{3} u^{3}$. We can determine the level sets of $E$ as shown in figure 5 and the local phase portraits as shown in figure 6 . The flow in the local chart $V_{3}$ is the same as that in the local chart $U_{3}$. Hence, the phase portrait of (1) on the sphere at infinity in the negative end point of the $z$-axis is shown in figure 6 , reversing time.

From the above analysis, we have the following proposition.

\section{PROPOSITION 2}

For any value of the parameter a, the phase portrait of system (1) on the Poincaré sphere at infinity is shown in figure 7. There are two cusps at the positive and negative ends of the z-axis and two nodes at the positive (stable) and negative (unstable) ends of the y-axis.

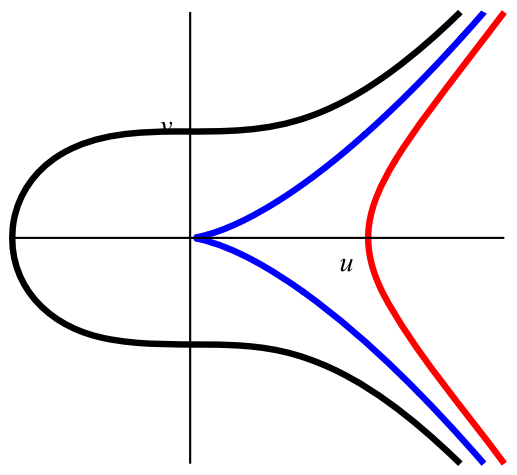

Figure 5. Level sets of total energy function $E$ for (8).

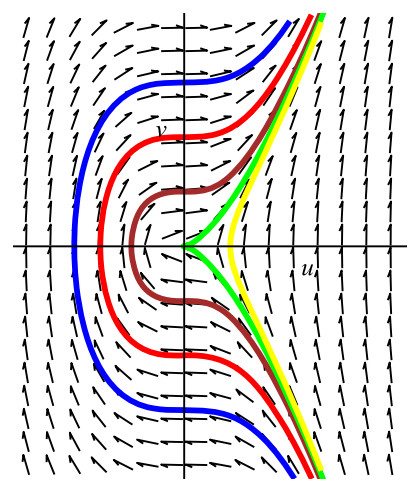

Figure 6. Local phase portraits near the cusp of (8).

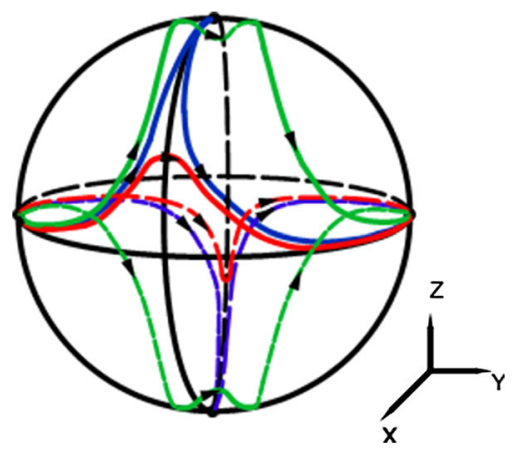

Figure 7. Phase portrait of system (1) on the Poincaré sphere at infinity.

\section{Existence of a Hopf bifurcation for system (1)}

According to Hopf bifurcation theory, substituting $\lambda=$ $\omega i$ in the characteristic equation, $f$,

$-\omega^{3} i-\omega^{2}+\left(8 a+\frac{1}{4}\right) \omega i+\frac{1}{4}=0$.

Separating real and imaginary parts, we obtain

$\left\{\begin{array}{l}-\omega^{3}+\left(8 a+\frac{1}{4}\right) \omega=0, \\ -\omega^{2}+\frac{1}{4}=0,\end{array}\right.$ 
leading to the following proposition:

\section{PROPOSITION 3}

The characteristic equation has a negative eigenvalue $\lambda_{1}=-1<0$ and a pair of purely imaginary eigenvalues $\lambda_{2,3}= \pm \frac{1}{2} i$ when $a=0$. System (1) then undergoes a Hopf bifurcation at the equilibrium point $P$.

Proof. If $a=0$, then the eigenvalues of system (1) at the equilibrium point $P$ are $\lambda_{1}=-1, \lambda_{2,3}= \pm \frac{1}{2} i$. Since

$$
\frac{\mathrm{d} \lambda}{\mathrm{d} a}=\frac{-8 \lambda}{3 \lambda^{2}+2 \lambda+\left(8 a+\frac{1}{4}\right)},
$$

the transversality condition satisfies:

$\operatorname{Re}\left(\left.\frac{\mathrm{d} \lambda}{\mathrm{d} a}\right|_{a=0}\right)=\operatorname{Re}\left(\left.\frac{-8 \lambda}{3 \lambda^{2}+2 \lambda+\frac{1}{4}}\right|_{\lambda= \pm \frac{1}{2} i}\right) \neq 0$

and system (2) undergoes a Hopf bifurcation at $O$. System (1) therefore undergoes a Hopf bifurcation at the point $P$.

\section{Stability of the bifurcating periodic solution}

In this section, we shall analyse the direction of the Hopf bifurcation and the stability of the bifurcating periodic solution of system (2) at $a=0$ using the centre manifold theorem. Let $x=X_{1}+\frac{1}{4}, y=Y_{1}+\frac{1}{16}, z=Z_{1}$, then eq. (1) can be transformed into

$$
\begin{aligned}
& \dot{X}_{1}=Y_{1} Z_{1}+\frac{1}{16} Z_{1}, \\
& \dot{Y}_{1}=X_{1}^{2}+\frac{1}{2} X_{1}-Y_{1}, \quad \dot{Z}_{1}=-4 X_{1} .
\end{aligned}
$$

The eigenvalues of the Jacobian matrix for system (9) are $\lambda_{1}=-1, \lambda_{2}=\frac{1}{2} i, \lambda_{3}=-\frac{1}{2} i$. The corresponding eigenvectors are

$$
\begin{aligned}
& v_{1}=\left(\begin{array}{l}
0 \\
1 \\
0
\end{array}\right), \quad v_{2}=\left(\begin{array}{c}
-\frac{1}{8} i \\
-\frac{1}{40}-\frac{1}{20} i \\
1
\end{array}\right), \\
& v_{3}=\left(\begin{array}{c}
\frac{1}{8} i \\
-\frac{1}{40}+\frac{1}{20} i
\end{array}\right) .
\end{aligned}
$$

Let

$$
u_{1}=\frac{\left(v_{2}+v_{3}\right)}{2}=\left(\begin{array}{c}
0 \\
-\frac{1}{40} \\
1
\end{array}\right) \text {, }
$$

$u_{2}=\frac{\left(v_{2}-v_{3}\right)}{2 i}=\left(\begin{array}{c}-\frac{1}{8} \\ -\frac{1}{20} \\ 0\end{array}\right)$,

$u_{3}=v_{1}=\left(\begin{array}{l}0 \\ 1 \\ 0\end{array}\right)$

and

$X_{1}=-\frac{1}{8} Y_{2}$,

$Y_{1}=-\frac{1}{40} X_{2}-\frac{1}{20} Y_{2}+Z_{2}$,

$Z_{1}=X_{2}$.

We then have

$$
\left\{\begin{array}{l}
\dot{X}_{2}=\frac{1}{2} Y_{2} \\
\dot{Y}_{2}=\frac{1}{5} X_{2}^{2}+\frac{2}{5} X_{2} Y_{2}-8 X_{2} Z_{2}-\frac{1}{2} X_{2} \\
\dot{Z}_{2}=\frac{1}{100} X_{2}^{2}+\frac{1}{50} X_{2} Y_{2}-\frac{2}{5} X_{2} Y_{2}+\frac{1}{64} Y_{2}^{2}-Z_{2}
\end{array}\right.
$$

Denote the local two-dimensional centre manifold of system (10) near the origin as the set

$W_{\mathrm{loc}}^{\mathrm{c}}(O)=\left\{\begin{array}{l|l}\left(X_{2}, Y_{2}, Z_{2}\right) \in R^{3} & \begin{array}{l}Z_{2}=h\left(X_{2}, Y_{2}\right), \\ \left|X_{2}\right|+\left|Y_{2}\right| \leq 1,\end{array}\end{array}\right\}$,

where

$h(0,0)=\frac{\partial h}{\partial X_{2}}(0,0)=\frac{\partial h}{\partial Y_{2}}(0,0)=0$.

Assuming $Z_{2}=h\left(X_{2}, Y_{2}\right)=a_{11} X_{2}^{2}+a_{12} X_{2} Y_{2}+$ $a_{22} Y_{2}^{2}+\cdots$, and denoting $X_{2}=w+u, Y_{2}=i(w-u)$, system (10) becomes

$$
\left\{\begin{aligned}
\dot{w}= & \frac{1}{2} i w-\frac{1}{10} i w^{2}-\frac{1}{5} i w u-\frac{1}{10} i u^{2} \\
& +\frac{1}{5} w^{2}-\frac{1}{5} u^{2}+4 i h w+4 i h u, \\
\dot{u}= & -\frac{1}{2} i u+\frac{1}{10} i w^{2}+\frac{1}{5} i w u+\frac{1}{10} i u^{2} \\
& -\frac{1}{5} w^{2}+\frac{1}{5} u^{2}-4 i h w-4 i h u, \\
\dot{Z}_{2}= & -\frac{9}{1600} w^{2}+\frac{41}{800} w u-\frac{9}{1600} u^{2} \\
& +\frac{1}{50} i w^{2}-\frac{1}{50} i u^{2}-\frac{2}{5} h w-\frac{2}{5} h u-h,
\end{aligned}\right.
$$

where $u=\bar{w}$. 
Rewriting $Z_{2}$ in the complex variable form:

$Z_{2}=N_{11} w^{2}+N_{12} w u+N_{22} u^{2}+o\left(|w|^{3}\right)$,

we have

$\dot{Z}_{2}=2 N_{11} \dot{w} w+N_{12}(\dot{w} u+w \dot{u})+2 N_{22} \dot{u} u$.

Substituting the first and the second equations of system (11) into system (12) gives

$$
\dot{Z}_{2}=i N_{11} w^{2}-i N_{12} u^{2}+o\left(|w|^{3}\right) \text {. }
$$

From the third equation of system (11), we have

$$
\begin{aligned}
\dot{Z}_{2}= & -\frac{9}{1600} w^{2}+\frac{41}{800} w u-\frac{9}{1600} u^{2}+\frac{1}{50} i w^{2} \\
& -\frac{1}{50} i u^{2}-\frac{2}{5} N_{11} w^{3}-\frac{2}{5} N_{12} u w^{2}-\frac{2}{5} N_{22} w u^{2} \\
& -\frac{2}{5} N_{11} u w^{2}-\frac{2}{5} N_{12} w u^{2}-\frac{2}{5} N_{22} u^{3}-N_{11} w^{2} \\
& -N_{12} w u-N_{22} u^{2} .
\end{aligned}
$$

Equating coefficients of $w^{2}, w u, u^{2}$ in (13) and (14), we have

$N_{11}=\frac{23}{3200}+i \frac{41}{3200}, \quad N_{22}=-\frac{23}{3200}-i \frac{41}{3200}$,

$N_{12}=-\frac{41}{800}$.

The dynamics on the centre manifold is then governed by the equation

$$
\begin{aligned}
\dot{w}= & \frac{1}{2} i w+\left(-\frac{1}{10} i+\frac{1}{5}\right) w^{2} \\
& -\frac{1}{5} i w u+\left(-\frac{1}{10} i-\frac{1}{5}\right) u^{2} \\
& +\left(-\frac{41}{800} i-\frac{41}{800}\right) u w^{2}+o\left(|w|^{3}\right) .
\end{aligned}
$$

Denoting the coefficients of $w^{2}, w u, u^{2}, w^{2} u$ as $g_{20}$, $g_{11}, g_{02}, g_{21}$ respectively, we obtain

$$
\begin{array}{ll}
g_{20}=2\left(-\frac{1}{10} i+\frac{1}{5}\right), & g_{11}=-\frac{1}{5} i, \\
g_{02}=2\left(-\frac{1}{10} i-\frac{1}{5}\right), & g_{21}=2\left(-\frac{41}{800} i-\frac{41}{800}\right) .
\end{array}
$$

The first Lyapunov coefficient is defined as

$\ell_{1}(0)=2 \operatorname{Re}(C(0))$,

where

$$
\begin{aligned}
C(0) & =i\left(g_{20} g_{11}-2\left|g_{11}\right|^{2}-\frac{1}{3}\left|g_{02}\right|^{2}\right)+\frac{g_{21}}{2} \\
& \simeq 2.875 \times 10^{-2}-0.2379 i .
\end{aligned}
$$

Since $\ell_{1}>0$, system (2) undergoes a subcritical Hopf bifurcation at the point $O$. We also have the following proposition which we state without proof.

\section{PROPOSITION 4}

System (1) undergoes a subcritical Hopf bifurcation at the equilibrium point $P$ when $a=0$. The point $P$ loses stability to an unstable limit cycle when $a>0$.

\section{Numerical simulations}

\subsection{Existence of the limit cycle}

In this section, we apply the results from the previous sections to system (1) for the Hopf bifurcation to show the existence of the limit cycle. Taking $a=0.01$, $a=0.03, a=0.05$ respectively, we obtain periodic orbits, as shown in figures 8,9 and 10 . The corresponding numerical values of the eigenvalues, Lyapunov

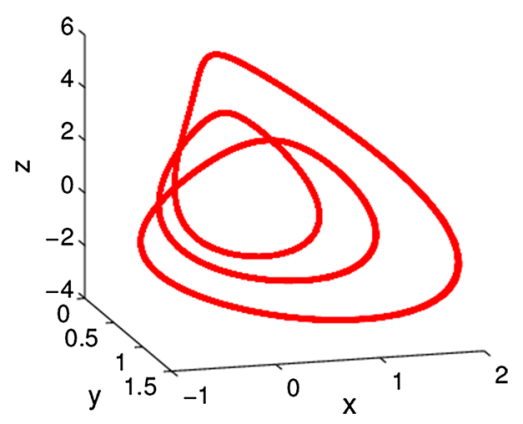

Figure 8. A periodic solution of period three for system (1) with $a=0.01$.

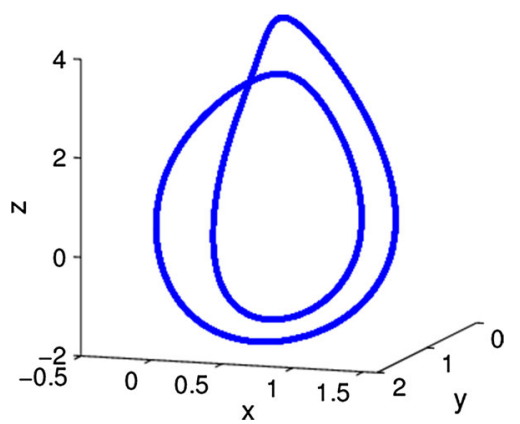

Figure 9. A periodic solution of period two for system (1) with $a=0.03$.

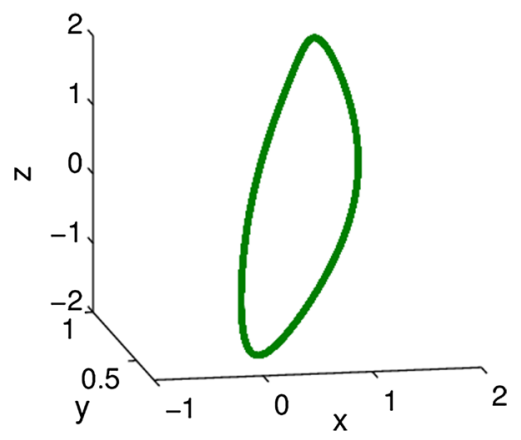

Figure 10. A periodic solution of period one (limit cycle) for system (1) with $a=0.05$. 
Table 1. Numerical results for three different parameter values for $a$. Here, Pv: Parameter value, Iv: Initial value, Ev: Eigenvalues, Le: Lyapunov exponents, Ld: Lyapunov dimension, Pp: Phase portrait.

\begin{tabular}{lccccc}
\hline $\mathrm{Pv}$ & $\mathrm{Iv}$ & $\mathrm{Ev}$ & $\mathrm{Le}$ & $\mathrm{Ld}$ & $\mathrm{Pp}$ \\
\hline$a=0.01$ & {$[1,1,1]$} & -0.93 & 0 & 1 & Figure 8 \\
& & $-0.03+0.52 i$ & -0.07 & & \\
& & $-0.03-0.52 i$ & -0.94 & & \\
$a=0.03$ & {$[1,1,1]$} & -0.78 & 0 & 1 & Figure 9 \\
& & $-0.11+0.55 i$ & -0.08 & & \\
& & $-0.11-0.55 i$ & -0.92 & & \\
$a=0.05$ & {$[1,1,1]$} & -0.61 & 0 & 1 & Figure 10 \\
& & $-0.20+0.61 i$ & -0.05 & & \\
& & $-0.20-0.61 i$ & -0.95 & & \\
\hline
\end{tabular}

Table 2. Numerical results for three different parameter values for $a$. Here, Pv: Parameter value, Iv: Initial value, Ev: Eigenvalues, Le: Lyapunov exponents, Ld: Lyapunov dimension, Pp: Phase portrait.

\begin{tabular}{|c|c|c|c|c|c|}
\hline $\mathrm{Pv}$ & $\mathrm{Iv}$ & $\mathrm{Ev}$ & Le & $\mathrm{Ld}$ & $\mathrm{Pp}$ \\
\hline$a=0.01$ & {$[1,1,3]$} & $\begin{array}{l}-0.93 \\
-0.03+0.52 i \\
-0.03-0.52 i\end{array}$ & $\begin{array}{l}0.06 \\
0 \\
-1.06\end{array}$ & 2.06 & Figure 11 \\
\hline$a=0$ & {$[0.1,0.01,-2.2]$} & $\begin{array}{r}-1 \\
0.5 i \\
-0.5 i\end{array}$ & $\begin{array}{l}0.08 \\
0 \\
-1.08\end{array}$ & 2.07 & Figure 12 \\
\hline$a=0$ & {$[0.26,0.0625,0.05]$} & $\begin{array}{l}-1 \\
0.5 i \\
-0.5 i\end{array}$ & $\begin{array}{r}0 \\
0 \\
-1\end{array}$ & 2 & Figure 13 \\
\hline
\end{tabular}

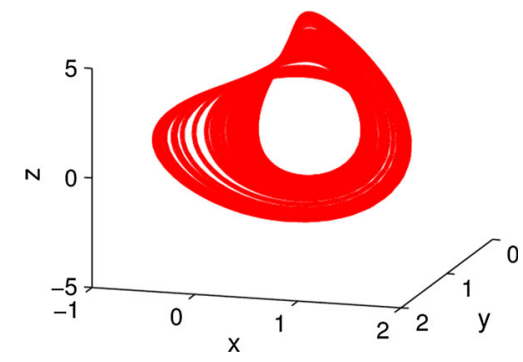

Figure 11. A chaotic attractor of system (1) with $a=0.01$.

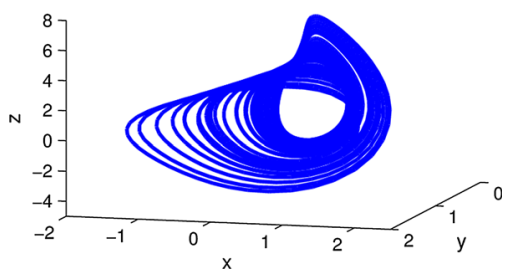

Figure 12. A chaotic attractor of system (1) with $a=0$.

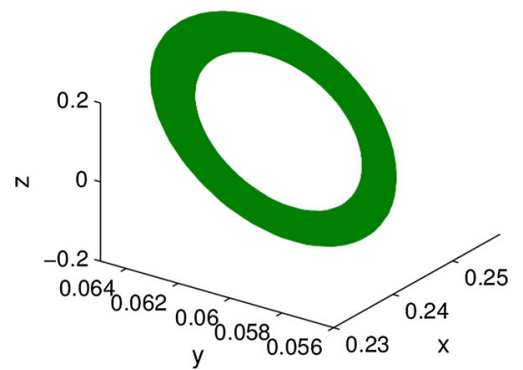

Figure 13. A quasiperiodic attractor of system (1) with $a=0$.

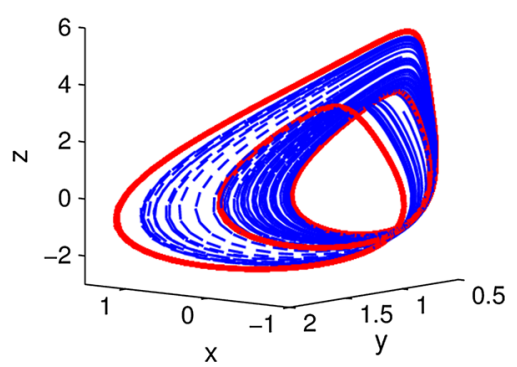

Figure 14. Coexisting attractors for system (1) with $a=0$. Dash line: chaotic attractor, real line: periodic attractor. 


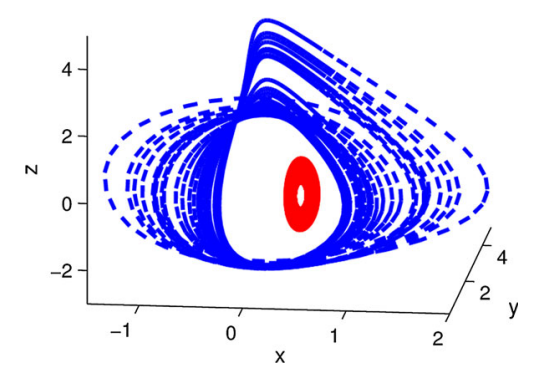

Figure 15. Coexisting attractors for system (1) with $a=0$, dash line: chaotic attractor, real line: quasiperiodic attractor.

exponents and Lyapunov dimensions are summarised in table 1.

\subsection{Coexisting attractors}

By choosing proper parameter values, many coexisting attractors of system (1) can be discovered by numerical integrations. The corresponding results are summarised in table 2, and the phase portraits are shown in figures 11,12 and 13 respectively. From tables 1 and 2, we see that when $a=0.01$, a chaotic attractor coexists with a period-three solution of system (1) (see figure 14), and when $a=0$, a chaotic attractor and a quasiperiodic attractor coexist, as shown in figure 15 .

\subsection{Basins of attraction}

In this subsection, we are interested in obtaining the basins of attraction of the different attracting sets defined as the set of initial points whose trajectories converge on the given attractor. Under the following linear transformation

$x_{1}=x-k_{1}, \quad y_{1}=y-k_{2}, \quad z_{1}=z-k_{3}$

system (1) becomes

$\left\{\begin{array}{l}\dot{x}_{1}=\left(y_{1}+k_{2}\right)\left(z_{1}+k_{3}\right)+a, \\ \dot{y}_{1}=\left(x_{1}+k_{1}\right)^{2}-\left(y_{1}+k_{2}\right), \\ \dot{z}_{1}=1-4\left(x_{1}+k_{1}\right) .\end{array}\right.$

The Hartman-Grobman theorem states that the local behaviour of an autonomous dynamical system in the neighbourhood of a hyperbolic equilibrium is qualitatively the same as (i.e. topologically equivalent to) the behaviour of its linearisation near this equilibrium. No matter how the values $k_{i}(i=1,2,3)$ change, the characteristic equations of system (16) and system (1) have the same eigenvalues at the corresponding equilibria. Consider the system parameter $a=0.01$ and $a=0$ respectively. System (16) (which is topologically equivalent to system (1)) has one stable equilibrium. Moreover, system (16) is chaotic for a certain parameter

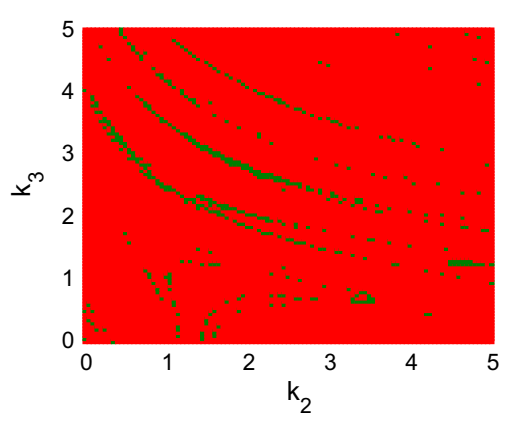

Figure 16. Dynamical region of system (16) with $a=0.01$ and $k_{1}=1$. Green regions denote non-chaotic states, while the red region denotes chaotic attractors.

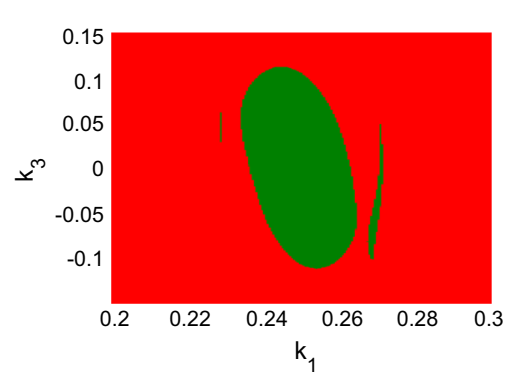

Figure 17. Dynamical region of system (16) with $a=0$ and $k_{2}=0.0625$. Green regions denote non-chaotic states, while the red region denotes chaotic attractors.

$k_{i}(i=1,2,3)$. Figures 16 and 17 show the dynamical regions in the $2 \mathrm{D}$ parameter spaces $\left(k_{2}-k_{3}\right)$ and $\left(k_{1}-k_{3}\right)$ of system (16) for $a=0.01$ and $a=0$. The green regions in the $\left(k_{2}-k_{3}\right)$ and $\left(k_{1}-k_{3}\right)$-planes correspond to non-chaotic states, while the red regions correspond to hidden chaotic attractors. For each point in this plot, it is necessary to search for initial conditions that give bounded solutions and then to estimate the largest Lyapunov exponent for each point. The criterion used is to assume that Lyapunov exponents in the range $(-0.001,0.001)$ are periodic (limit cycles, shown in green), while those that are more negative correspond to stable equilibria (point attractors, shown in green), and those that are more positive correspond to chaotic attractors (hidden attractors, shown in red).

\subsection{Bifurcation analysis}

When $a=0$, system (1) is the Sprott E system, and it degenerates in the sense that its Jacobian eigenvalues at the equilibria consist of one conjugate pair of pure imaginary numbers and one real number. The Shilnikov homoclinic criterion might not be applied to this system. When $a>0$, the stability of the equilibrium is fundamentally different from that of the Sprott E system. In this case, the equilibrium becomes a node-focus. The 


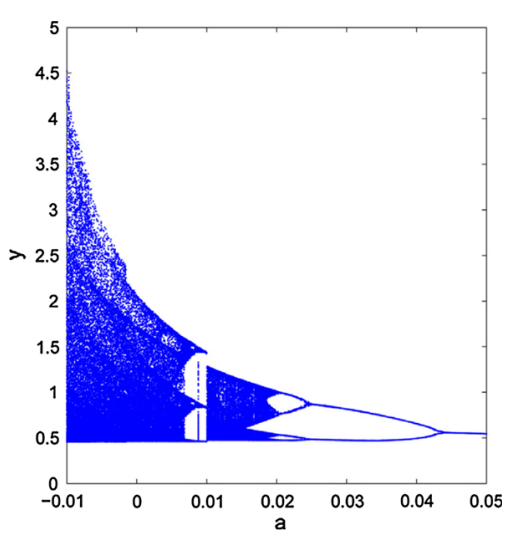

Figure 18. Bifurcation diagram, showing a period-halving bifurcation (reversed period bifurcation) route to chaos in $y$ (at $x=0.25$ ) vs. the parameter $a$.

Shilnikov homoclinic criterion is therefore inapplicable to this case. Moreover, according to the definition of self-excited attractor and hidden attractor (an attractor is called a self-excited attractor if its basin of attraction intersects with any open neighbourhood of a stationary state (an equilibrium), otherwise it is called a hidden attractor) [32], hidden attractors are attractors in systems with no equilibria or with only one stable equilibrium, and the nature of the attractor of system (1) is a hidden attractor. Figure 18 shows a bifurcation diagram versus the parameter $a$, demonstrating a reverse period doubling route to chaos, and indicates that although the equilibrium is changed from an unstable saddle-focus to a stable node-focus, the chaotic dynamics survive in a relative narrow range of the parameter $a$.

\section{Conclusion}

In this paper, the dynamics at infinity of Sprott E system perturbed with a tiny constant $a$ is analysed using Poincaré-Lyapunov compactification. The Hopf bifurcation for this system is computed by the theory of centre manifolds, and a subcritical Hopf bifurcation is obtained. Numerical simulations confirmed the correctness of the Hopf bifurcation analysis. Choosing appropriate parameters, this perturbated system can exhibit chaotic, quasiperiodic and periodic dynamics. In particular, coexisting attractors can be observed, such as a chaotic attractor and a periodic attractor for $a>0$ and a chaotic attractor and a quasiperiodic attractor for $a=0$.

Another form of complexity arises for asymptotically stable equilibrium points for attracting sets coexisting via $a>0$. This is usually referred to as coexisting attractors and when this occurs, the trajectories of the system selectively converge on either of the attracting sets depending on the initial state of the system. When coexisting attractors occur, engineers and scientists are usually interested in obtaining the basins of attraction of the different attracting sets, defined as the set of initial points whose trajectories converge on the given attractor [25]. Indeed, the global dynamical behaviours and the geometrical structure of the current system have not been presented completely. The generation mechanism of the chaos for this special system, as well as the algebraic structure (or normal form) for this system need to be studied. These will be provided in future works.

\section{Acknowledgements}

The author acknowledges the referees and the editor for carefully reading this paper and suggesting many helpful comments. This work was supported by the Natural Science Foundation of China (No. 11726624), the Natural Science Basic Research Plan in Shaanxi Province of China (No. 2016JM1024), the Natural Science Basic Research Plan in Shandong Province of China (No. ZR2017PA008), Shaanxi Key Laboratory of Complex System Control and Intelligent Information Processing (No. 2016CP06), Xi' an University of Technology and the Scientific Research Foundation of Xijing University (No. XJ160142).

\section{References}

[1] E N Lorenz, J. Atmos. Sci. 20(2), 130 (1963)

[2] G R Chen and T Ueta, Int. J. Bifurc. Chaos Appl. Sci. Engng. 9(7), 1465 (1999)

[3] S Celikovsky and A Vanecek, Kybernetika 30(4), 403 (1994)

[4] J H Lü and G R Chen, Int. J. Bifurc. Chaos Appl. Sci. Engng. 12(3), 659 (2002)

[5] Q G Yang, Z C Wei and G R Chen, Int. J. Bifurc. Chaos Appl. Sci. Engng. 20(4), 1061 (2010)

[6] Z C Wei and Q G Yang, Appl. Math. Comput. 217(1), $422(2010)$

[7] Z C Wei and Q G Yang, Nonlinear Anal. Real World Appl. 12(1), 106 (2011)

[8] J C Sprott, Phys. Rev. E 50(2), 647 (1994)

[9] J C Sprott, Phys. Lett. A 228(4-5), 271 (1997)

[10] J C Sprott, Am. J. Phys. 65(6), 537 (1997)

[11] X Wang and G R Chen, Commun. Nonlinear Sci. Numer. Simul. 17(3), 1264 (2012)

[12] Z C Wei and Z Wang, Kybernetika 49(2), 359 (2013)

[13] M Molaie, S Jafari, J C Sprott and S Mohammad Reza Hashemi Golpayegani, Int. J. Bifurc. Chaos Appl. Sci. Engng. 23(11), 1350188 (2013)

[14] S T Kingni, S Jafari, H Simo and P Woafo, Eur. Phys. J. Plus 129(5), 1 (2014) 
[15] S K Lao, Y Shekofteh, S Jafari and J C Sprott, Int. J. Bifurc. Chaos Appl. Sci. Engng. 24(1), 1450010 (2014)

[16] Z C Wei, Phys. Lett. A 376(2), 102 (2011)

[17] S Jafari, J C Sprott and S Mohammad, Phys. Lett. A 377(9), 699 (2013)

[18] X Wang and G R Chen, Nonlinear Dynam. 71(3), 429 (2013)

[19] S Jafari and J C Sprott, Chaos Solitons Fractals 57(12), 79 (2013)

[20] G A Leonov and N V Kuznetsov, Dokl. Math. 84(1), 475 (2011)

[21] G A Leonov, N V Kuznetsov, O A Kuznetsov, S M Seledzhi and V I Vagaitsev, Trans. Syst. Control 6(2), 54 (2011)

[22] G A Leonov and N V Kuznetsov, Physica D 241(18), 1482 (2012)

[23] Z C Wei, W Zhang, Z Wang and M H Yao, Int. J. Bifurc. Chaos Appl. Sci. Engng. 25(02), 1550028 (2015)

[24] Z C Wei, J C Sprott and H Chen, Phys. Lett. A 379(37), 2184 (2015)
[25] D Dudkowskia, S Jafari, T Kapitaniak, N V Kuznetsov, G A Leonov and A Prasad, Phys. Rep. 637(1), 1 (2016)

[26] Z Wang, W Sun, Z C Wei and X J Xi, Kybernetika 50(4), 616 (2014)

[27] M Abramowitz and I A Stegun, Handbook of mathematical functions with formulas, graphs, and mathematical tables (Dover, Washington, 1965)

[28] F Dumortier, J Llibre and J C Artes, Qualitative theory of planar differential systems (Springer, Berlin, 2006)

[29] Z Wang, Z C Wei, X J Xi and Y X Li, Nonlinear Dynam. 77(4), 1503 (2014)

[30] Y J Liu and Q G Yang, Int. J. Bifurc. Chaos Appl. Sci. Engng. 21(9), 2559 (2011)

[31] R C Robinson, An introduction to dynamical systems: Continuous and discrete (American Mathematical Society, New York, 2012)

[32] G A Leonov, N V Kuznetsov and T N Mokaev, Eur. Phys. J. Spec. Top. 224(8), 1421 (2015) 